\title{
Locally recoverable codes from automorphism group of function fields of genus $\mathbf{g} 1$
}

\author{
Bartoli, Daniele; Montanucci, Maria; Quoos, Luciane
}

Published in:

IEEE Transactions on Information Theory

Link to article, DOI:

10.1109/TIT.2020.2995852

Publication date:

2020

Document Version

Peer reviewed version

Link back to DTU Orbit

Citation (APA):

Bartoli, D., Montanucci, M., \& Quoos, L. (2020). Locally recoverable codes from automorphism group of function fields of genus g 1. IEEE Transactions on Information Theory, 66(11), 6799 - 6808.

https://doi.org/10.1109/TIT.2020.2995852

\section{General rights}

Copyright and moral rights for the publications made accessible in the public portal are retained by the authors and/or other copyright owners and it is a condition of accessing publications that users recognise and abide by the legal requirements associated with these rights.

- Users may download and print one copy of any publication from the public portal for the purpose of private study or research.

- You may not further distribute the material or use it for any profit-making activity or commercial gain

- You may freely distribute the URL identifying the publication in the public portal

If you believe that this document breaches copyright please contact us providing details, and we will remove access to the work immediately and investigate your claim. 


\title{
Locally recoverable codes from automorphism group of function fields of genus $g \geq 1$
}

\author{
Daniele Bartoli, Maria Montanucci, and Luciane Quoos
}

\begin{abstract}
A Locally Recoverable Code is a code such that the value of any single coordinate of a codeword can be recovered from the values of a small subset of other coordinates. When we have $\delta$ non-overlapping subsets of cardinality $r_{i}$ that can be used to recover the missing coordinate we say that a linear code $\mathcal{C}$ with length $n$, dimension $k$, minimum distance $d$ has $\left(r_{1}, \ldots, r_{\delta}\right)$ locality and denote by $\left[n, k, d ; r_{1}, r_{2}, \ldots, r_{\delta}\right]$. In this paper we provide a new upper bound for the minimum distance of these codes. Working with a finite number of subgroups of cardinality $r_{i}+1$ of the automorphism group of a function field $\mathcal{F} \mid \mathbb{F}_{q}$ of genus $g \geq 1$ we propose a construction of $\left[n, k, d ; r_{1}, r_{2}, \ldots, r_{\delta}\right]$ codes and apply the results to some well known families of function fields.
\end{abstract}

Index Terms-Codes for distributed storage; Locally Recoverable Codes; Hamming distance; covering maps; maximal curves.

\section{INTRODUCTION}

The study of Locally Recoverable codes (LRC for short) was motivated by the use of coding theory techniques applied to distributed and cloud storage systems. Local recovery techniques enable the repair of lost encoded data by a local procedure, that is by making use of small amount of data instead of all information contained in a codeword. Formally, a LRC code of length $n$ is a code that produces an $n$-symbol codeword from $k$ information symbols and, for any symbol of the codeword, there exist at most $r$ other symbols such that the value of the symbol can be recovered from them. This value $r$ is the called the locality of the code. For example, a code of length $2 k$ in which each coordinate is repeated twice, is an LRC code with locality $r=1$. Generally the locality parameter satisfies $1 \leq r \leq k$ since the entire codeword can be found by accessing $k$ symbols other than the erased symbol.

In distributed storage systems, erasure codes with locality $r$ are preferred because a coordinate can be locally repaired by accessing at most $r$ other coordinates. However, the local

Daniele Bartoli is with the Dipartimento di Matematica e Informatica, Università degli Studi di Perugia, Via Vanvitelli 1 - 06123 Perugia - Italy, email: daniele.bartoli@unipg.it

Maria Montanucci is with the Department of Applied Mathematics and Computer Science, Technical University of Denmark, Kongens Lyngby 2800, Denmark,email: marimo@dtu.dk

Luciane Quoos is with the Instituto de Matemática, Federal University of Rio de Janeiro, Centro de Tecnologia - Bloco C, Cidade Universitária - Av. Athos da Silveira Ramos, 149 - Ilha do Fundão, CEP 21.941-909 - Brazil, email: luciane@im.ufrj.br repair may not be performed when some of the $r$ coordinates are also erased. To overcome this problem, we can work with $\delta$ non-overlapping local repair sets of size no more than $r_{i}$ for a coordinate. The formal definition of a Locally Recoverable Code is given in Definition II.1. We denote a linear code $\mathcal{C}$ with length $n$, dimension $k$, minimum distance $d$, and $\left(r_{1}, \ldots, r_{\delta}\right)$-locality by $\left[n, k, d ; r_{1}, r_{2}, \ldots, r_{\delta}\right]$.

In recent years, the study of locally reparable codes has attracted a lot of attention. Most of the results concern bounds on the minimum distance [9], [18], [27], [28] and construction of LRC codes [5], [11], [14]-[16], [19], [20], [22], [23], [26], [28].

The goal of this paper is twofold. First we present a new upper bound on the minimum distance of a $\left[n, k, d ; r_{1}, r_{2}, \ldots, r_{\delta}\right]$-code. Second we present two constructions of LRCs over function fields $\mathcal{F} \mid \mathbb{F}_{q}$ of genus $g \geq 1$ using subgroups of their automorphism group. We apply these constructions on some well known families of curves with many rational points.

Let $\mathcal{C}$ be an $\left[n, k, d ; r_{1}, \ldots, r_{\delta}\right]$-code. If $\delta=1$ it was proved in 2012 that the minimum distance of the code is upper bounded by

$$
d \leq n-k-\left\lceil\frac{k}{r}\right\rceil+2
$$

see [9]. This bound coincides with the classical Singleton bound when $r=k$. A code that achieves equality in (1) is called an optimal LRC code. In [19], [20], [26] the authors constructed optimal LRC codes using particular types of polynomials, cyclic codes, and elliptic curves respectively.

For general $\delta \geq 1$ and $r=r_{1}=\cdots=r_{\delta}$ the bound (1) was generalized in 2014 to

$$
d \leq n-\sum_{i=0}^{t}\left\lfloor\frac{k-1}{r^{i}}\right\rfloor
$$

see [26].

Since in our paper we deal with general $\left[n, k, d ; r_{1}, \ldots, r_{\delta}\right]$ codes, in order to check the quality of the constructed codes, exploring ideas from [28] we first generalize (1) to

$$
d \leq n-k-\left\lceil\frac{(k-1) \delta+1}{1+\sum_{i=1}^{\delta} r_{i}}\right\rceil+2
$$


see Theorem III.2. In analogy with the definition of the singleton defect for linear codes, a notion of relative defect for $\left[n, k, d ; r_{1}, \ldots, r_{\delta}\right]$-code is introduced using the bound in (3) as

$$
\Delta(\mathcal{C})=\frac{1}{n}\left(n-k-d+2-\left\lceil\frac{(k-1) \delta+1}{1+\sum_{i=1}^{\delta} r_{i}}\right\rceil\right) .
$$

Intuitively, the smaller the relative defect of a code, the better the code.

In this work we also provide two general constructions of LRC codes with locality $\left(r_{1}, \ldots, r_{\delta}\right)$ over function fields $\mathcal{F} \mid \mathbb{F}_{q}$ of genus $g \geq 1$ using a finite number of subgroups of cardinality $r_{i}+1$ of the automorphism group $\operatorname{Aut}\left(\mathcal{F} \mid \mathbb{F}_{q}\right)$. We distinguish the cases of trivial intersection (Theorem IV.1) and nontrivial intersection (Theorem V.1) and we provide a generalization of the construction proposed in [15] for the rational function field.

It is well known that maximal function fields and function fields with many rational points provide algebraic geometry codes with "good" parameters; see e.g. [2]-[4], [13]. We build up several families of LRC codes applying Theorems IV.1 and V.1 to families of maximal function fields, such as the Hermitian [6], the Giulietti-Korchmáros [8], the generalized Hermitian [10], [17], and the Norm-Trace [24] ones. In particular, we obtain families of locally repairable codes with good relative parameters, since the relative defect $\Delta(\mathcal{C})$ as in (4) tends to zero when $q$ goes to infinity; see Propositions IV.2, IV.4, IV.6, IV.8, and IV.9. Also, we compare the parameters of some of these LRC codes with the ones constructed in [11] obtained from fiber products of algebraic curves; see Remark IV.7.

The paper is organized as follows. In Section 2, we present some preliminaries on locally repairable codes, function fields, automorphism groups, and algebraic geometry codes. In Section 3 a new upper bound for the minimum distance $d$ of a $\left[n, k, d ; r_{1}, r_{2}, \ldots, r_{\delta}\right]$-code depending on the locality $\left(r_{1}, r_{2}, \ldots, r_{\delta}\right)$ is provided; see Theorem III.2. This bound is used to define the relative defect $\delta$ of such code, a parameter that will be used to measure how good a code is. In Sections 3 and 4 we present two types of constructions of locally repairable codes via subgroups of the automorphism group of a function field. In Theorem IV.1 we deal with subgroups with trivial intersection, and in Theorem V.1 the case of nontrivial intersection is investigated. In the same sections we apply the constructions to some well known function fields with many rational places.

\section{PRELIMINARIES}

Consider an $[n, k, d]$-code $\mathcal{C}$ over $\mathbb{F}_{q}$ and generator matrix $\left(g_{1}, \ldots, g_{n}\right)$. For any subset $I \subset\{1, \ldots, n\}$ let $\left\langle g_{j}: j \in I\right\rangle$ denote the $\mathbb{F}_{q}$-subspace generated by the set $\left\{g_{j}: j \in I\right\}$.
The code $\mathcal{C} \subseteq \mathbb{F}_{q}^{n}$ is said to have locality $\left(r_{1}, r_{2}, \ldots, r_{\delta}\right)$ if the value of every coordinate of a given codeword can be retrieved by accessing any of the $\delta$ disjoint recovering sets of cardinalities $r_{1}, r_{2}, \ldots, r_{\delta}$. One error in the codeword can be corrected in a local way if no more than $\delta-1$ erasures occur. A formal definition of a locally repairable code is given as follows.

Definition II.1. [1] The $i$-th coordinate, where $1 \leq i \leq$ $n$, of an $[n, k, d]$ linear code $\mathcal{C}$ whose generator matrix is $\left(g_{1}, \ldots, g_{n}\right)$ is said to have $\left(r_{1}, \ldots, r_{\delta}\right)$-locality if there exist pairwise disjoint repair sets $R_{1}^{(i)}, \ldots, R_{\delta}^{(i)} \in\{1, \ldots, n\} \backslash\{i\}$ such that for each $1 \leq j \leq \delta$

i) $\# R_{j}^{(i)}=r_{i}$;

ii) $g_{i} \in\left\langle g_{\ell} \mid \ell \in R_{j}^{(i)}\right\rangle$.

Note that $r_{i}=1$ implies repetition and we only consider codes with $\delta \geq 1$ and $r_{i} \geq 2$. Additionally, we always assume $r_{i}<k$. We denote a linear code $\mathcal{C}$ with length $n$, dimension $k$, minimum distance $d$, and with $\left(r_{1}, \ldots, r_{\delta}\right)$-locality by

$$
\left[n, k, d ; r_{1}, r_{2}, \ldots, r_{\delta}\right] \text {. }
$$

A set $I \subset\{1, \ldots, n\}$ is called an information set for an $\left[n, k, d ; r_{1}, r_{2}, \ldots, r_{\delta}\right]$-code if $\# I=\operatorname{rank}\left(\left\langle g_{j} \mid j \in I\right\rangle\right)=k$

We now fix some notation and collect some results on function fields and algebraic geometry codes; for more on this subject we refer to the book [25]. Given a function field $\mathcal{F} \mid \mathbb{F}_{q}$ and a function $z \in \mathcal{F}$ let $(z)_{\infty}$ be its pole divisor. For a divisor $D$ in $\mathcal{F}$ the $\mathbb{F}_{q}$-vector space

$$
\mathcal{L}(D)=\left\{z \in \mathcal{F}:(z)_{\infty} \geq-D\right\} \cup\{0\}
$$

is the Riemann-Roch space associated to $D$ and its dimension is denoted by $\ell(D)$. Let

$$
\begin{gathered}
\operatorname{Aut}\left(\mathcal{F} \mid \mathbb{F}_{q}\right)=\{\sigma: \mathcal{F} \mapsto \mathcal{F}: \sigma \text { is an automorphism } \\
\text { of } \left.\mathcal{F} \text { and } \sigma(a)=a, \forall a \in \mathbb{F}_{q}\right\}
\end{gathered}
$$

be the automorphism group of the function field $\mathcal{F} \mid \mathbb{F}_{q}$. For a subgroup $\mathcal{H}$ of $\operatorname{Aut}\left(\mathcal{F} \mid \mathbb{F}_{q}\right)$ we let $\mathcal{F}^{\mathcal{H}}$ denote the fixed field of $\mathcal{H}$. The group $A u t\left(\mathcal{F} \mid \mathbb{F}_{q}\right)$ acts on set of rational places of the function field in a natural way and, for any rational place $P \in \mathcal{F}$ and any subgroup $\mathcal{H}$ of $\operatorname{Aut}\left(\mathcal{F} \mid \mathbb{F}_{q}\right)$, we let $P^{\mathcal{H}}=\{\sigma(P): \sigma \in \mathcal{H}\}$ stand for the orbit of $P$ under the action of $\mathcal{H}$. The orbit is either short or long provided $P^{\mathcal{H}}<\mathcal{H}$ or $P^{\mathcal{H}}=\mathcal{H}$ respectively.

Fixing $\mathcal{H}$ and $P \in \mathcal{F}^{\mathcal{H}}$ an $\mathbb{F}_{q^{-}}$rational place, the ramification of $P$ in the Galois extension $\mathcal{F} \mid \mathcal{F}^{\mathcal{H}}$ is determined by the action of $\mathcal{H}$ on the orbit $P_{1}^{\mathcal{H}}$ where $P_{1}$ is any fixed $\mathbb{F}_{q^{-}}$ rational place over $P$. In particular, $P$ is totally ramified if and only if $P_{1}^{\mathcal{H}}=\left\{P_{1}\right\}$, and $P$ is completely split if and only if $\# P_{1}^{\mathcal{H}}=\# \mathcal{H}$ and every place $P_{1}$ above $P$ is $\mathbb{F}_{q}$-rational.

Let $G$ be a divisor on $\mathcal{F}$ and $P_{1}, P_{2}, \ldots, P_{n}$ be pairwise distinct rational places on $\mathcal{F}$, with $P_{i} \notin \operatorname{supp}(G)$ for all $i$. 
Define $D=\sum_{i=1}^{n} P_{i}$. The linear algebraic geometry code $\mathcal{C}_{\mathcal{L}}(D, G)$ is defined as the image of the evaluation function

$$
\text { ev }: \mathcal{L}(G) \rightarrow \mathbb{F}_{q^{n}}, f \mapsto\left(f\left(P_{1}\right), f\left(P_{2}\right), \ldots, f\left(P_{n}\right)\right) .
$$

The $\mathcal{C}_{\mathcal{L}}(D, G)$ code has length $n$ and the classical bound on the minimum distance $d$ is

$$
d \geq n-\operatorname{deg}(G) .
$$

\section{A BOUND ON THE MINIMUM DISTANCE}

Let $\mathcal{C}$ be an $\left[n, k, d ; r_{1}, \ldots, r_{\delta}\right]$-code. Locally recoverable (LRC-)codes attaining equality in (1) are called optimal. Constructions of optimal LRC-codes on curves of genus one can be found in [19], and with minimum distance 3 and 4 were obtained using cyclic codes in [20].

In [28, Theorem 1] another upper bound on the minimum distance was derived for the case $\delta \geq 1$ and $r=r_{1}=\cdots=r_{\delta}$

$$
d \leq n-k+2-\left\lceil\frac{(k-1) \delta+1}{(r-1) \delta+1}\right\rceil .
$$

In what follows we generalize the ideas in [28] in order to provide a new bound on the minimum distance of a general $\left[n, k, d ; r_{1}, \ldots, r_{\delta}\right]$-code. First we recall a well known lemma about the minimum distance of a code.

Lemma III.1. [21] The minimum distance of any $[n, k, d]$ code whose generator matrix is $\left(g_{1}, \ldots, g_{n}\right)$ satisfies

$d=n-\max \left\{\# N: N \subset\{1, \ldots, n\}, \operatorname{rank}\left(\left\langle g_{j} \mid j \in N\right\rangle\right)<k\right\}$.

Theorem III.2. For an $\left[n, k, d ; r_{1}, \ldots, r_{\delta}\right]_{q}$ linear code, with $2 \leq r_{1} \leq r_{2} \leq \cdots \leq r_{\delta}<k$,

$$
d \leq n-k+2-\left\lceil\frac{(k-1) \delta+1}{1+\sum_{i=1}^{\delta} r_{i}}\right\rceil .
$$

Proof. Let $I \subset\{1, \ldots, n\}$ be an information set such that each coordinate in $I$ has $\left(r_{1}, \ldots, r_{\delta}\right)$-locality. For any $i \in$ $I$ and $j=1, \ldots, \delta$ let $R_{j}^{(i)}$ be the repair set of cardinality $\# R_{j}^{(i)}=r_{i}$, and $1 \leq a \leq \delta$ denote by $N_{a}^{(i)}$ the set $\{i\} \cup$ $R_{1}^{(i)} \cup \cdots \cup R_{a}^{(i)}$. So,

$$
\operatorname{rank}\left(\left\langle g_{j}: j \in N_{a}^{(i)}\right\rangle\right) \leq 1+\sum_{i=1}^{a} r_{i} .
$$

Since $I$ is an information set there exists $\left\{i_{1}, \ldots, i_{\ell}\right\} \subset I$ such that

$$
\operatorname{rank}\left(\left\langle g_{j}: j \in \bar{N}\right\rangle\right)=k-1,
$$

where

$$
\bar{N}=N_{\delta}^{\left(i_{1}\right)} \cup \cdots \cup N_{\delta}^{\left(i_{\ell-1}\right)} \cup R_{1}^{\left(i_{\ell}\right)} \cup \cdots \cup R_{\theta}^{\left(i_{\ell}\right)} \cup \bar{R},
$$

with $0 \leq \theta<\delta, \bar{R} \subset R_{\theta+1}^{\left(i_{\ell}\right)}$. Denote by $X$ and $Y$ the quantities $\sum_{i=1}^{\delta} r_{i}$ and $\sum_{i=1}^{\theta+1} r_{i}$ respectively. From (8) we have

$$
\begin{aligned}
k-1 \leq & \operatorname{rank}\left(\left\langle g_{j}: j \in \bar{N}\right\rangle\right) \\
\leq & \sum_{r=1}^{\ell-1} \operatorname{rank}\left(\left\langle g_{j}: j \in N_{\delta}^{\left(i_{r}\right)}\right\rangle\right) \\
& \quad+\sum_{r=1}^{\theta+1} \operatorname{rank}\left(\left\langle g_{j}: j \in R_{r}^{\left(i_{\ell}\right)}\right\rangle\right) \\
\leq & (\ell-1)(1+X)+Y,
\end{aligned}
$$

that is

$$
\ell-1 \geq\left\lceil\frac{k-1-Y}{1+X}\right\rceil .
$$

Since $g_{i_{r}} \in \bigcap_{a=1}^{\delta}\left\langle g_{j}: j \in R_{a}^{\left(i_{r}\right)}\right\rangle$, the difference $\# N_{a}^{i_{r}}-$ $\operatorname{rank}\left(\left\langle g_{j}: j \in N_{a}^{i_{r}}\right\rangle\right)$ is at least $a$.

Therefore,

$$
\begin{aligned}
\# \bar{N} & \geq \operatorname{rank}\left(\left\langle g_{j}: j \in \bar{N}\right\rangle\right)+(\ell-1) \delta+\theta \\
& \geq k-1+\left\lceil\frac{k-1-Y}{1+X}\right\rceil \delta+\theta \\
& \geq k-1+\frac{k-1-Y}{1+X} \delta+\theta \\
& =k-2+\frac{(k-1) \delta+1}{1+X}+\frac{(1+X)(1+\theta)-1-Y \delta}{1+X} .
\end{aligned}
$$

Consider now $(1+X)(1+\theta)-1-Y \delta$.

- Suppose first that $\theta=\delta-1$. Then $(1+X)(1+\theta)-1-$ $Y \delta=(1+X) \delta-1-Y \delta \geq \delta-1$, since $X \geq Y$.

- If $\delta \geq \theta+2$, then $(1+X)(1+\theta)-1-Y \delta=$

$$
\begin{aligned}
& X(\theta+1)-Y \delta+\theta \\
= & (\theta+1) \sum_{i=1}^{\theta+1} r_{i}+(\theta+1) \sum_{i=\theta+2}^{\delta} r_{i}-\delta \sum_{i=1}^{\theta+1} r_{i}+\theta \\
& -(\delta-\theta-1) \sum_{i=1}^{\theta+1} r_{i}+(\theta+1) \sum_{i=\theta+2}^{\delta} r_{i}+\theta \\
\geq & -(\delta-\theta-1)(\theta+1) r_{\theta+1} \\
& +(\theta+1)(\delta-\theta-1) r_{\theta+2}+\theta \\
\geq & \theta .
\end{aligned}
$$

So, $(1+X)(1+\theta)-1-Y \delta \geq 0$. Since $\# \bar{N}$ is an integer,

$$
\# \bar{N} \geq k-2+\left\lceil\frac{(k-1) \delta+1}{1+X}\right\rceil .
$$

By Lemma III.1,

$$
\begin{aligned}
n= & d+\max \{\# N: N \subset\{1, \ldots, n\}, \\
& \left.\operatorname{rank}\left(\left\langle g_{j}: j \in N\right\rangle\right)<k\right\} \geq d+\# \bar{N} \\
\geq & d+k-2+\left\lceil\frac{(k-1) \delta+1}{1+X}\right\rceil,
\end{aligned}
$$

and the claim follows. 
Remark III.3. Note that for $\delta=1$, Bound (7) is weaker than Bound (1).

In [15] the authors construct LRC codes over the rational function field $\mathbb{F}_{q}(x)$ (genus zero) dealing with subgroup of its automorphism group, and obtain codes with length $n \simeq q$ roughly and relative defect $\simeq 1 / q$ according to Formula (4).

\section{General CONSTRUCTION FROM SUbGROUPS With TRIVIAL INTERSECTION}

Many constructions of LRC codes from function fields arose in recent years, over the rational function field [14], [15], elliptic function fields [19] and over algebraic curves (see [22], [28]), fiber product of curves [11] and curves with separated variables [23]. In a variant of the different ways to construct LRC codes, we focus on codes from algebraic function fields of genus $g \geq 1$ using certain subgroups of the automorphism group of the underlying curve. In this section we deal with the case of subgroups with trivial intersection and in the next Section with non-trivial intersection subgroups.

Theorem IV.1. Let $\mathcal{F} \mid \mathbb{F}_{q}$ be a function field of genus $g$. Consider s subgroups $\mathcal{H}_{i}$ of the automorphism group of $\mathcal{F} \mid \mathbb{F}_{q}$, each of sizes $r_{i}+1$, such that the group $\mathcal{G} \simeq \bigotimes_{i=1}^{s} \mathcal{H}_{i}$ is isomorphic to the internal direct product of $\mathcal{H}_{1}, \ldots, \mathcal{H}_{s}$. Let $\mathcal{P}$ be a set of places in $\mathcal{F}$ lying over $m$ rational places in the fixed field $\mathcal{F}^{\mathcal{G}}$ that are completely split in the extension $\mathcal{F} \mid \mathcal{F}^{\mathcal{G}}$. Define $n=m \prod_{i=1}^{s}\left(r_{i}+1\right)$, that is, $n$ to be the total number of places of $\mathcal{F}$ lying over the $m$ selected rational places in $\mathcal{F}^{\mathcal{G}}$.

Suppose that there exists a place $P_{\infty}$ of $\mathcal{F}$ which is completely ramified in $\mathcal{F} \mid \mathcal{F}^{\mathcal{G}}$ and let $Q_{\infty}^{(i)}$ be the unique place in $\mathcal{F}^{\mathcal{H}_{i}}$ lying under $P_{\infty}$. For $i=1, \ldots, s$ suppose further there exist functions $z_{i}, w_{i}$, such that

i) $z_{i} \in \mathcal{F}^{\mathcal{H}_{i}}, \operatorname{supp}\left(\left(z_{i}\right)_{\infty}\right)=\left\{Q_{\infty}^{(i)}\right\}$;

ii) $w_{i} \in \mathcal{F} \backslash \mathcal{F}^{\mathcal{H}_{i}}, \operatorname{supp}\left(\left(w_{i}\right)_{\infty}\right)=\left\{P_{\infty}\right\}$;

iii) $w_{i}: P^{\mathcal{H}_{i}} \rightarrow \mathbb{F}_{q}$ is injective.

For each $i=1, \ldots, s$ let $t_{i} \geq 1$ be such that

$$
V_{i}:=\left\{\sum_{\ell=0}^{r_{i}-1}\left(\sum_{j=0}^{t_{i}} a_{\ell j}^{(i)} z_{i}^{j}\right) w_{i}^{\ell} \in \mathcal{F} \mid a_{\ell j}^{(i)} \in \mathbb{F}_{q}\right\}
$$

is contained in $\mathcal{L}\left((n-d) P_{\infty}\right)$ for some $1 \leq d \leq n$ and let $V=\bigcap_{i=1}^{s} V_{i} \subset \mathcal{L}\left((n-d) P_{\infty}\right)$. If $\operatorname{dim}_{\mathbb{F}_{q}}(V)>0$, then there exists an

$$
\left[n, \operatorname{dim}_{\mathbb{F}_{q}}(V), \geq d ; r_{1}, \ldots, r_{s}\right] \text {-recoverable code. }
$$

Proof. Let $V=\bigcap_{i=1}^{s} V_{i} \subset \mathcal{L}\left((n-d) P_{\infty}\right)$ and consider the following linear map

$$
\begin{aligned}
e_{\mathcal{P}}: \quad V & \rightarrow \mathbb{F}_{q}^{n} \\
f & \mapsto e_{\mathcal{P}}(f)=\left(f\left(P_{1}\right), \ldots, f\left(P_{n}\right)\right),
\end{aligned}
$$

where $\mathcal{P}=\left\{P_{1}, \ldots, P_{n}\right\}$.

The linear code $e_{\mathcal{P}}(V)$ is contained in the algebraic geometry code $C_{\mathcal{L}}\left(\sum_{i}^{n} P_{i},(n-d) P_{\infty}\right)$. Then the minimum distance of the code $e_{\mathcal{P}}(V)$ is larger than or equal to the minimum distance of the linear code $C_{\mathcal{L}}\left(\sum_{i}^{n} P_{i},(n-d) P_{\infty}\right)$. By the bound on Equation (5), the code $C_{\mathcal{L}}\left(\sum_{i}^{n} P_{i},(n-d) P_{\infty}\right)$ has minimum distance at least $d$. By definition it has length $n$ and dimension $\operatorname{dim}_{\mathbb{F}_{q}}(V)$.

Now, we deal with recoverability. First note that a rational place $Q \in \mathcal{F}^{\mathcal{G}}$ which is completely split in $\mathcal{F} \mid \mathcal{F}^{\mathcal{G}}$ satisfies $P^{\mathcal{H}_{i}} \cap P^{\mathcal{H}_{j}}=\{P\}$ for each $i \neq j \in\{1, \ldots, s\}$, where $P$ is an arbitrary place in $\mathcal{P}$ lying over $Q$. In fact, since $Q$ is completely split, by the orbit stabilizer theorem, this means that the stabilizer in $G$ of $P$ is trivial. If $P^{\mathcal{H}_{i}}$ and $P^{\mathcal{H}_{j}}$ contain another common place $\tilde{P} \neq P$ then there exist $h_{i} \in \mathcal{H}_{i}$ and $h_{j} \in \mathcal{H}_{j}$ such that $\tilde{P}=h_{i}(P)=h_{j}(P)$ so that $h_{i} h_{j}^{-1}(P)=$ $P$. Since $\mathcal{H}_{i} \cap \mathcal{H}_{j}$ is trivial, $h_{i} h_{j}^{-1}$ is a non-trivial element of $\mathcal{G}$, a contradiction. Consider a fixed place $P \in \mathcal{P}$ and an $f \in V$. We are going to show that $f(P)$ can be repaired by any of the $s$ recoverable sets (depending on $P$ )

$$
R_{i}=\left\{f\left(P^{\prime}\right): P^{\prime} \in P^{\mathcal{H}_{i}} \backslash\{P\}\right\}, \text { for }
$$

of cardinality $r_{i}$ for $i=1, \ldots, s$. Since $f \in V_{i}$ we can write $f=\sum_{\ell=0}^{r_{i}-1}\left(\sum_{j=0}^{t_{i}} a_{\ell j}^{(i)} z_{i}^{j}\right) w_{i}^{\ell}, a_{\ell j}^{(i)} \in \mathbb{F}_{q}$. Since $z_{i} \in \mathcal{F}^{\mathcal{H}_{i}}$ we have that $z_{i}\left(Q_{1}\right)=z_{j}\left(Q_{2}\right)=\bar{z}$ for any $Q_{i}, Q_{j} \in P^{\mathcal{H}_{i}}$, then for each $Q \in P^{\mathcal{H}_{i}}$ we have

$$
\begin{aligned}
f(Q) & =\sum_{\ell=0}^{r_{i}-1}\left(\sum_{j=0}^{t_{i}} a_{\ell j}^{(i)} z_{i}^{j}(Q)\right) w_{i}(Q)^{\ell} \\
& =\sum_{\ell=0}^{r_{i}-1}\left(\sum_{j=0}^{t_{i}} a_{\ell j}^{(i)} \bar{z}^{j}\right) w_{i}(Q)^{\ell} \\
& =\sum_{\ell=0}^{r_{i}-1} \gamma_{\ell, P}^{(i)} w_{i}(Q)^{\ell},
\end{aligned}
$$

where $\gamma_{\ell, P}^{(i)} \in \mathbb{F}_{q}$ depends only on $\ell$ (and $P$ clearly). So,

$$
h(X)=\sum_{\ell=0}^{r_{i}-1} \gamma_{\ell, P}^{(i)} X^{\ell} \in \mathbb{F}_{q}[X]
$$

is a polynomial of degree at most $r_{i}-1$. Since the values $w_{i}(Q)$ with $Q \in P^{\mathcal{H}_{i}}$ are distinct we know exactly $r_{i}$ different values of $h(X)$. By Lagrange interpolation we can recover $h(X)$ and then compute the missing value $h\left(w_{i}(P)\right)$. By definition of $\mathcal{P}$, the recovering sets $P^{\mathcal{H}_{i}} \backslash\{P\}$ are pairwise disjoint.

In what follows we are going to construct examples of LRC codes over algebraic function fields of genus $g \geq 1$ based on Theorem IV.1. All the constructed codes comes from algebraic curves with many rational points. 


\section{A. Codes from the Hermitian Function Field I}

Consider the Hermitian function field $\mathcal{F}=\mathbb{F}_{q^{2}}(x, y)$ with $y^{q+1}=x^{q}+x$. Choose a divisor $u \geq 2$ of $q+1$. Consider the following two subgroups of $\mathbb{F}_{q}$-automorphisms of $\mathcal{F}$

$$
\begin{aligned}
\mathcal{H}_{1}:= & \left\{(x, y) \mapsto(x+c, y) \mid c \in \mathbb{F}_{q^{2}}, c^{q}+c=0\right\}, \\
\mathcal{H}_{2}:= & \left\{(x, y) \mapsto\left(x, a^{i} y\right) \mid i=0, \ldots, u-1\right. \text { and } \\
& \left.a \in \mathbb{F}_{q^{2}}^{*} \text { of order } u \geq 2\right\} .
\end{aligned}
$$

It is easy to see that $\# \mathcal{H}_{1}=q, \# \mathcal{H}_{2}=u, \mathcal{F}^{\mathcal{H}_{1}}=\mathbb{F}_{q^{2}}(y)$ and $\mathcal{F}^{\mathcal{H}_{2}}=\mathbb{F}_{q^{2}}\left(x, y^{u}\right)$. The groups have trivial intersection and commute, then $\mathcal{G}=\mathcal{H}_{1} \mathcal{H}_{2}=\mathcal{H}_{1} \times \mathcal{H}_{2}$ and $\mathcal{F}^{\mathcal{G}}=\mathbb{F}_{q^{2}}(z:=$ $\left.y^{u}\right) \subseteq \mathcal{F}^{\mathcal{H}_{i}}$, for $i=1,2$. Let $P_{\infty}$ be the unique pole of $x$ and $y \in \mathcal{F}$, then $(y)_{\infty}=q P_{\infty}$ and $(x)_{\infty}=(q+1) P_{\infty}$ in $\mathcal{F}$. Denote by $Q_{\infty}^{i}=P_{\infty} \cap \mathcal{F}^{\mathcal{H}_{i}}$, for $i=1,2$. In the extension $\mathcal{F} \mid \mathbb{F}_{q^{2}}(z)$, we have that $\left(q^{2}-1\right) / u$ places in $\mathcal{F}^{\mathcal{G}}$ are totally split, so \#P $=q\left(q^{2}-1\right)=n$. For $t_{i} \geq 2, i=1,2$ consider the $\mathbb{F}_{q}$-vector spaces

$$
\begin{aligned}
V_{1} & :=\left\{\sum_{\ell=0}^{q-2}\left(\sum_{j=0}^{t_{1}} a_{\ell, j} y^{j}\right) x^{\ell}: a_{\ell, j} \in \mathbb{F}_{q^{2}}\right\} \text { and } \\
V_{2} & :=\left\{\sum_{\ell=0}^{u-2}\left(\sum_{j=0}^{t_{2}} a_{\ell, j} z^{j}\right) y^{\ell}: a_{\ell, j} \in \mathbb{F}_{q^{2}}\right\} \\
& =\left\{\sum_{\ell=0}^{u-2} \sum_{j=0}^{t_{2}} a_{\ell, j} y^{(u-1) j+\ell}: a_{\ell, j} \in \mathbb{F}_{q^{2}}\right\} .
\end{aligned}
$$

Note that $\operatorname{dim}_{\mathbb{F}_{q^{2}}} V_{1}=(q-1)\left(t_{1}+1\right)$ and $\operatorname{dim}_{\mathbb{F}_{q^{2}}} V_{2}=$ $(u-1)\left(t_{2}+1\right)$. A function in $V_{1}$ has pole only at the place $P_{\infty}$ of order at most $q^{2}-q-2+t_{1} q$, while a function in $V_{2}$ also has pole only at $P_{\infty}$ of order at most $q\left((u-1) t_{2}+u-2\right)$.

Using the notations of the Theorem IV.1, we have $z_{1}=$ $y, z_{2}=y^{u-1}, w_{1}=x$ and $w_{2}=y$. Now we choose the parameters:

i) $t_{1}=q-1$ and $\frac{2 q^{2}-q u-2}{q(u-1)} \leq t_{2}<\frac{q^{2}}{u-1}-1$ so that

$$
\left.V_{1}, V_{2} \subset \mathcal{L}\left(q\left((u-1) t_{2}+u-2\right)\right) P_{\infty}\right),
$$

and $d=n-q\left(u t_{2}+u-t_{2}-2\right) \geq 1$.

Now,

$$
V=V_{1} \cap V_{2}=\left\{\sum_{j=0}^{q-1} a_{j} y^{j}: a_{j} \in \mathbb{F}_{q^{2}}\right\}
$$

and $\operatorname{dim}_{\mathbb{F}_{q^{2}}} V=q$.

ii) $u\left(t_{2}+1\right) \stackrel{q^{2}}{=} q+1, t_{1} \geq 1,\left(t_{1}, t_{2}\right) \neq(1,1)$, then

$$
V_{1}, V_{2} \subset \mathcal{L}\left(\left(q^{2}-q-2+t_{1} q\right) P_{\infty}\right),
$$

and $V=V_{1} \cap V_{2}=\left\{\sum_{j=0}^{q-1} a_{j} y^{j}: a_{j} \in \mathbb{F}_{q^{2}}\right\}$.

The two choices of parameters in the construction can be summarized as the following.
Proposition IV.2. For every $1<u$ a divisor of $q+1$ and $\frac{2 q^{2}-q u-2}{q(u-1)} \leq t_{2}<\frac{q^{2}}{u-1}-1$ there exists a

$\left[q\left(q^{2}-1\right), q, \geq q\left(q^{2}-1\right)-q\left(u t_{2}+u-t_{2}-2\right) ; q-1, u-1\right]$

recoverable code $\mathcal{C}_{1}$.

For every $1<u, u\left(t_{2}+1\right)=q+1, t_{1} \geq 2$ and $\left(t_{1}, t_{2}\right) \neq$ $(1,1)$ there exists a

$\left[q\left(q^{2}-1\right), q, \geq q\left(q^{2}-1\right)-\left(q^{2}-q-2+t_{1} q\right) ; q-1, u-1\right]$

recoverable code $\mathcal{C}_{2}$.

Remark IV.3. The relative defect of the codes $\mathcal{C}_{1}$ and $\mathcal{C}_{2}$ (see Equation (4)) are

$$
\begin{aligned}
\Delta\left(\mathcal{C}_{1}\right) & \leq \frac{1}{q\left(q^{2}-1\right)}\left(q\left(u t_{2}+u-t_{2}-2\right)-q+2\right. \\
& \left.-\left\lceil\frac{2 q-1}{q+u-1}\right\rceil\right)=\frac{q\left(u t_{2}+u-t_{2}-3\right)+1}{q\left(q^{2}-1\right)}, \\
\Delta\left(\mathcal{C}_{2}\right) & \leq \frac{1}{q\left(q^{2}-1\right)}\left(\left(q^{2}-q-2+t_{1} q\right)-q+2\right. \\
& \left.-\left\lceil\frac{2 q-1}{q+u-1}\right\rceil\right)=\frac{q^{2}-2 q+t_{1} q-1}{q\left(q^{2}-1\right)} \simeq \frac{q+t_{1}}{q^{2}-1} .
\end{aligned}
$$

For the code $\mathcal{C}_{1}$, if $t_{2}$ is close to the lower bound $\frac{2 q^{2}-q u-2}{q(u-1)}$, $\Delta\left(\mathcal{C}_{1}\right)$ is less or equal to $2 / q$.

\section{B. Codes from the Hermitian Function Field II}

Consider the Hermitian function field $\mathcal{F}=\mathbb{F}_{q^{2}}(x, y)$ with $y^{q+1}=x^{q}+x$. Use the same notations as in Section IV-A. Consider now

$$
\begin{aligned}
V_{1} & :=\left\{\sum_{\ell=0}^{q-1}\left(\sum_{j=0}^{t_{1} \leq u} a_{\ell, j} y^{j}\right) x^{\ell}: a_{\ell, j} \in \mathbb{F}_{q^{2}}\right\}, \\
V_{2} & :=\left\{\sum_{\ell=0}^{u-1}\left(\sum_{j=0}^{t_{2} \leq q} a_{\ell, j} x^{j}\right) y^{\ell}: a_{\ell, j} \in \mathbb{F}_{q^{2}}\right\},
\end{aligned}
$$

of dimensions $\operatorname{dim}_{\mathbb{F}_{q^{2}}} V_{1}=\left(t_{1}+1\right) q$ and $\operatorname{dim}_{\mathbb{F}_{q^{2}}} V_{2}=\left(t_{2}+\right.$ 1) $u$.

A function in $V_{1}$ has pole only at the place $P_{\infty}$ of order at most $q^{2}-q-2+t_{1} q$, while a function in $V_{2}$ also has pole only at $P_{\infty}$ of order at most $q\left((u-1) t_{2}+u-2\right)$.

Using the notations of the Theorem IV.1 we have $z_{1}=y$, $z_{2}=x, w_{1}=x, w_{2}=y$. Also,

$$
V=V_{1} \cap V_{2}=\left\{\sum_{\ell=0}^{t_{1}}\left(\sum_{j=0}^{t_{2}} a_{\ell, j} x^{j}\right) y^{\ell}: a_{\ell, j} \in \mathbb{F}_{q^{2}}\right\}
$$

and $\operatorname{dim}_{\mathbb{F}_{q^{2}}} V=\left(t_{1}+1\right)\left(t_{2}+1\right)$. For

$$
M=\max \left\{t_{1} q+q^{2}-1,(u-1) q+t_{2}(q+1)\right\} \leq 2 q^{2}+q-1
$$


we have $V \subset \mathcal{L}\left(M P_{\infty}\right)$. In this case $n-d=M$ and $d \geq$ $\max \left\{q^{2}+t_{1}\left(q-t_{2}\right),(u-1) q+t_{2}\left(q-t_{1}\right)\right\}$. We obtain the following proposition.

Proposition IV.4. For every divisor $1<u$ of $q+1,1 \leq t_{1} \leq$ $u, 1 \leq t_{2} \leq q$ and

$$
M=\max \left\{t_{1} q+q^{2}-1,(u-1) q+t_{2}(q+1)\right\}
$$

there exists a

$$
\left[q\left(q^{2}-1\right),\left(t_{1}+1\right)\left(t_{2}+1\right), \geq q\left(q^{2}-1\right)-M ; q-1, u-1\right]
$$

recoverable code $\mathcal{C}$.

Remark IV.5. The relative defect of this code satisfies

$$
\Delta(\mathcal{C}) \leq \frac{M-\left(t_{1}+1\right)\left(t_{2}+1\right)+2-\left\lceil\frac{2\left(t_{1} t_{2}+t_{1}+t_{2}\right)+1}{q+u-1}\right\rceil}{q\left(q^{2}-1\right)} .
$$

Choosing $u=q+1, t_{1}=u$ and $t_{2}=q$ we obtain $\Delta(\mathcal{C}) \leq$ $\frac{q^{2}-q-1}{q\left(q^{2}-1\right)}$ and so the relative defect goes to zero as $q$ goes to infinity.

\section{Codes from the Giulleti-Korchmáros curve}

Let $\mathcal{F}=\mathbb{F}_{q^{6}}(x, y, z)$ be the function field of the curve $\mathcal{G K}$ whose affine model is given by the complete intersection

$$
\left\{\begin{array}{l}
Z^{q^{2}-q+1}=Y^{q^{2}}-Y \\
Y^{q+1}=X^{q}+X
\end{array}\right.
$$

This is a maximal curve over $\mathbb{F}_{q^{6}}$ and has $q^{8}-q^{6}+q^{5}+1$ rational places and only one place $P_{\infty}$ at infinity. Moreover, $P_{\infty}$ is the common pole of $x, y$ and $z$ with pole divisors

$(x)_{\infty}=\left(q^{3}+1\right) P_{\infty},(y)_{\infty}=\left(q^{3}-q^{2}+q\right) P_{\infty},(z)_{\infty}=q P_{\infty}$,

see [8]. In this case we consider three subgroups of the following types, let $A$ be a subgroup of $\left\{a \in \mathbb{F}_{q^{6}}: a^{q}+a=0\right\}$, $\eta, \omega \in \mathbb{F}_{q^{6}}$, with $\operatorname{ord}(\eta) \mid q^{3}+1$, $\operatorname{ord}(\omega) \mid q^{2}-q+1$, and $\operatorname{gcd}(\operatorname{ord}(\eta), \operatorname{ord}(\omega))=1$. Consider the following subgroups of $\operatorname{Aut}\left(\mathcal{F} \mid \mathbb{F}_{q^{6}}\right)$ :

$$
\begin{aligned}
\mathcal{H}_{1}:= & \left\{\sigma_{a}:(x, y, z) \mapsto(x+a, y, z): a \in A\right\} ; \\
\mathcal{H}_{2}:= & \left\{\sigma_{i}:(x, y, z) \mapsto\left(x, \eta^{i\left(q^{2}-q+1\right)} y, \eta^{i} z\right):\right. \\
& i=0, \ldots, \operatorname{ord}(\eta)-1\} ; \\
\mathcal{H}_{3}:= & \left\{\sigma_{i}:(x, y, z) \mapsto\left(x, y, \omega^{i} z\right):\right. \\
& i=0, \ldots, \operatorname{ord}(\omega)-1\},
\end{aligned}
$$

Then we have $\mathcal{G}=\mathcal{H}_{1} \mathcal{H}_{2} \mathcal{H}_{3} \cong \mathcal{H}_{1} \times \mathcal{H}_{2} \times \mathcal{H}_{3}$ is a subgroup of $\operatorname{Aut}\left(\mathcal{F} \mid \mathbb{F}_{q^{6}}\right)$. The fixed fields satisfy $\mathbb{F}_{q^{6}}(y, z)=$ $\mathcal{F}^{\mathcal{H}_{1}}, \mathbb{F}_{q^{6}}(x) \subseteq \mathcal{F}^{\mathcal{H}_{2}}$ and $\mathbb{F}_{q^{6}}(x, y) \subseteq \mathcal{F}^{\mathcal{H}_{3}}$. Now we notice that for all $P=(x, y, z)$ a rational place with $z \neq 0$ we have that $P^{\mathcal{H}_{i}} \cap P^{\mathcal{H}_{j}}=\{P\}, i, j \in\{1,2,3\}$. In fact, if $Q \in P^{\mathcal{H}_{2}} \cap P^{\mathcal{H}_{3}}$, since the unique possibility for $\eta^{i}=1$ and $\omega^{j}=1$ yields $i=j=0$ from $\operatorname{gcd}(\operatorname{ord}(\eta), \operatorname{ord}(\omega))=1$, we obtain $Q=P$. The other cases are trivial. Therefore, all the affine $\mathbb{F}_{q^{6}}$-rational points of $\mathcal{G K}$ belong to long orbits with respect to $\mathcal{G}$ are those with $z \neq 0$. So, the cardinality of the set $\mathcal{P}$ as in Theorem IV.1 can be taken as $n=q^{8}-q^{6}+q^{5}-q^{3}=q^{3}\left(q^{2}-1\right)\left(q^{3}+1\right)$. Using the notations in Theorem IV. 1 we consider

i) $z_{1}:=z \in \mathcal{F}^{\mathcal{H}_{1}}, w_{1}:=x \in \mathcal{F} \backslash \mathcal{F}^{\mathcal{H}_{1}}$

ii) $z_{2}:=x \in \mathcal{F}^{\mathcal{H}_{2}}, w_{2}:=z \in \mathcal{F} \backslash \mathcal{F}^{\mathcal{H}_{2}}$

iii) $z_{3}:=x \in \mathcal{F}^{\mathcal{H}_{3}}, w_{1}:=z \in \mathcal{F} \backslash \mathcal{F}^{\mathcal{H}_{3}}$.

For any integers $t_{i} \geq 0, i=1,2,3$, consider the following $\mathbb{F}_{q^{6} \text {-vector spaces }}$

$$
\begin{aligned}
& V_{1}:=\left\{\sum_{\ell=0}^{\# A-2}\left(\sum_{j=0}^{t_{1}} a_{\ell, j} z^{j}\right) x^{\ell}: a_{\ell, j} \in \mathbb{F}_{q^{6}}\right\}, \\
& V_{2}:=\left\{\sum_{\ell=0}^{\operatorname{ord}(\eta)-2}\left(\sum_{j=0}^{t_{2}} a_{\ell, j} x^{j}\right) z^{\ell}: a_{\ell, j} \in \mathbb{F}_{q^{6}}\right\}, \text { and } \\
& V_{3}:=\left\{\sum_{\ell=0}^{\operatorname{ord}(\omega)-2}\left(\sum_{j=0}^{t_{3}} a_{\ell, j} x^{j}\right) z^{\ell}: a_{\ell, j} \in \mathbb{F}_{q^{6}}\right\} .
\end{aligned}
$$

For $N_{1}=\min \left\{\# A-2, t_{2}, t_{3}\right\}, M_{1}=\min \left\{t_{1}, \operatorname{ord}(\eta)-\right.$ $2, \operatorname{ord}(\omega)-2\}$ and

$$
\begin{aligned}
S= & \max \left\{t_{2}\left(q^{3}+1\right)+(\operatorname{ord}(\eta)-2) q,\right. \\
& \left.(\# A-2)\left(q^{3}+1\right)+t_{1} q, t_{3}\left(q^{3}+1\right)+(\operatorname{ord}(\omega)-2) q\right\},
\end{aligned}
$$

we obtain that

$V:=V_{1} \cap V_{2} \cap V_{3}=\left\{\sum_{n_{1}=0}^{N_{1}} \sum_{m_{1}=0}^{M_{1}} a_{n_{1}, m_{1}} z^{m_{1}} x^{n_{1}}: a_{n_{1}, m_{1}} \in \mathbb{F}_{q^{6}}\right\}$

is contained in $\mathcal{L}\left(S P_{\infty}\right)$ and it has dimension $\operatorname{dim}_{\mathbb{F}_{q^{6}}} V=$ $\left(M_{1}+1\right)\left(N_{1}+1\right)$. Choosing $t_{i}, i=1,2,3$ such that $q^{8}-$ $q^{6}+q^{5}-q^{3}-S \geq 1$ we obtain the following proposition.

Proposition IV.6. Let $q=p^{\ell}, p$ prime. Then for any

i) $a=p^{h}, 1 \leq h \leq \ell$,

ii) $\operatorname{ord}(\eta)\left|q^{3}+1, \operatorname{ord}(\omega)\right| q^{2}-q+1$, with $\operatorname{gcd}(\operatorname{ord}(\eta), \operatorname{ord}(\omega))=1$,

iii) $0<t_{1}<q^{2}\left(q^{2}-1\right)\left(q^{3}+1\right)-q^{3}+2 q^{2}-1,0<t_{2}<$ $q^{3}\left(q^{2}-1\right)-q, 0<t_{3}<q^{3}\left(q^{2}-1\right)-1$,

iv) $N_{1}=\min \left\{a-2, t_{2}, t_{3}\right\}, M_{1}=\min \left\{t_{1}, \operatorname{ord}(\eta)-\right.$ $2, \operatorname{ord}(\omega)-2\}$, and

v) $S=\max \left\{(a-2)\left(q^{3}+1\right)+t_{1} q, t_{2}\left(q^{3}+1\right)+(\operatorname{ord}(\eta)-\right.$ 2) $\left.q, t_{3}\left(q^{3}+1\right)+(\operatorname{ord}(\omega)-2) q\right\}$,

there exists a

$\left[n,\left(M_{1}+1\right)\left(N_{1}+1\right), \geq n-S ; a-1, \operatorname{ord}(\eta)-1, \operatorname{ord}(\omega)-1\right]$

recoverable code $\mathcal{C}_{3}$ over $\mathbb{F}_{q^{6}}$, where $n=q^{8}-q^{6}+q^{5}-q^{3}$. 
Its relative defect $\Delta\left(\mathcal{C}_{3}\right)$ is at most

$$
\frac{S-\left(M_{1}+1\right)\left(N_{1}+1\right)+2-\left\lceil\frac{3\left(M_{1} N_{1}+M_{1}+N_{1}\right)+1}{a+\operatorname{ord}(\eta)+\operatorname{ord}(\omega)-2}\right\rceil}{q^{8}-q^{6}+q^{5}-q^{3}} .
$$

Suppose that $\left(q^{3}+1\right)$ possesses two coprime divisors both close to $q \sqrt{q}$. Consider $q \sqrt{q}<t_{1}<q^{2}, t_{2}, t_{3} \simeq a$, so that $N_{1}=a-2, M_{1} \simeq q \sqrt{q}, S \simeq N_{1} q^{3}$ and therefore $\Delta\left(\mathcal{C}_{3}\right)$ is at most a value close to $N_{1}\left(q^{3}-q \sqrt{q}\right) / q^{8}$.

Remark IV.7. In [11] the authors consider fiber products of algebraic curves in order to construct LRC codes. In particular [11, Corollary 1] can be seen as a construction from trivially intersecting subgroups of the automorphism group of an algebraic curve. In what follows we choose codes with same length and compare the obtained relative defects.

In [11, Theorem 5.1] the authors construct codes on the generalized GK curve. For the special case of the curve GK with $\ell=q$, they obtain $a$

$\left[n,\left(q^{2}-q\right)\left(q^{2}-1\right), n-q^{5}+2 q^{3}-q^{2}-q+2 ; q-1, q^{2}-q\right]$ recoverable code over $\mathbb{F}_{q^{6}}$, where $n=q^{8}-q^{6}+q^{5}-q^{3}$.

The relative defect of such a code is $\frac{q^{5}-q^{4}-q^{3}+2 q+1}{n}$.

There are many choices for $a, \eta, \omega$ such that the code constructed from Proposition IV.6 has relative defect smaller than $\frac{q^{5}-q^{4}-q^{3}+2 q+1}{n}$. Suppose $3 \nmid(q+1)$. Consider for instance $a=q, \operatorname{ord}(\eta)=q+1, \operatorname{ord}(\omega)=q^{2}-q+1$, $t_{1}=t_{2}=t_{3}=q-1$. In this case, $N_{1}=q-2$, $M_{1}=q-1$ and $S=q^{4}-q^{2}-1$. Note that $S$ is the maximum $S=\max \left\{(a-2)\left(q^{3}+1\right)+t_{1} q, t_{2}\left(q^{3}+1\right)+(\operatorname{ord}(\eta)-\right.$ 2) $\left.q, t_{3}\left(q^{3}+1\right)+(\operatorname{ord}(\omega)-2) q\right\}=t_{3}\left(q^{3}+1\right)+(\operatorname{ord}(\omega)-2) q=$ $(q-1)\left(q^{3}+1\right)+\left(q^{2}-q-1\right) q=q^{4}-q^{2}-1$, and hence there exists an

$$
\left[n, \geq q^{8}-q^{6}+q^{5}-q^{4}-q^{3}+q^{2}+1 ; q-1, q, q^{2}-q\right]
$$

recoverable code $\mathcal{C}_{3}$ over $\mathbb{F}_{q^{6}}$, where $n=q^{8}-q^{6}+q^{5}-$ $q^{3}, q(q-1)$, whose relative defect is at most

$$
\begin{aligned}
& \frac{S-\left(M_{1}+1\right)\left(N_{1}+1\right)+2-\left\lceil\frac{3\left(M_{1} N_{1}+M_{1}+N_{1}\right)+1}{a+\operatorname{ord}(\eta)+\operatorname{ord}(\omega)-2}\right\rceil}{n} \\
& \leq \frac{q^{4}-2 q^{2}+q}{n} .
\end{aligned}
$$

Similar results can be obtained when $3 \mid(q+1)$ by considering for instance $\operatorname{ord}(\eta)=q+1$ and $\operatorname{ord}(\omega)=\left(q^{2}-q+1\right) / 3$. Also in this case, the relative defect is roughly $q^{4} / n$.

\section{Codes from the generalized Hermitian curve}

Let $q$ be odd. The curve $y^{q^{\ell}+1}=x^{q}+x$ over $\mathbb{F}_{q^{2 \ell}}$ with $\ell \geq$ 1 odd has $q^{2 \ell+1}+1$ rational points and genus $g=q^{\ell}(q-1) / 2$, see [10], [17]. This curve has only one rational point at infinity denoted by $P_{\infty}$, it is also the only pole of the functions $x$ and $y$ with pole order $q^{\ell}+1$ and $q$, respectively. Let $\mathcal{S}$ be the function field of the curve $y^{q^{\ell}+1}=x^{q}+x$, and consider two subgroups of the automorphism group of $\mathcal{S}$, given by

$$
\begin{aligned}
\mathcal{H}_{1}:= & \left\{(x, y) \mapsto(x+a, y) \mid a^{q}+a=0 \text { and } a \in \mathbb{F}_{q^{2 \ell}}\right\}, \\
& \text { and } \\
\mathcal{H}_{2}:= & \left\{(x, y) \mapsto(x, \lambda y) \mid \lambda \in \mathbb{F}_{q^{2 \ell}} \text { and } \lambda^{q^{\ell}+1}=1\right\},
\end{aligned}
$$

so that $\# \mathcal{H}_{1}=q, \# \mathcal{H}_{2}=q^{\ell}+1$ and the fixed fields are $\mathcal{S}^{\mathcal{H}_{1}}=\mathbb{F}_{q^{2 \ell}}(y), \mathcal{S}^{\mathcal{H}_{2}}=\mathbb{F}_{q^{2 \ell}}(x)$, and $\mathcal{S}^{\mathcal{G}}=\mathbb{F}_{q^{2 \ell}}\left(x^{q}+x\right)=$ $\mathbb{F}_{q^{2 \ell}}\left(y^{q^{\ell}+1}\right)$ by the direct product $\mathcal{G} \simeq \mathcal{H}_{1} \times \mathcal{H}_{2}$. The number of rational places in $\mathcal{S}^{\mathcal{G}}$ that completely splits in the field extension $\mathcal{S} \mid \mathcal{S}^{\mathcal{G}}$ is $q^{\ell}-1$, then we have $n=\# \mathcal{P}=q^{2 \ell+1}-q$. Let $0 \leq t_{i}, i=1,2$ and consider the vector spaces

$$
\begin{aligned}
V_{1} & :=\left\{\sum_{\ell=0}^{q-2}\left(\sum_{j=0}^{t_{1}} a_{\ell, j} y^{j}\right) x^{\ell}: a_{\ell, j} \in \mathbb{F}_{q^{2 \ell}}\right\}, \\
V_{2} & :=\left\{\sum_{\ell=0}^{q^{\ell}-1}\left(\sum_{j=0}^{t_{2}} a_{\ell, j} x^{j}\right) y^{\ell}: a_{\ell, j} \in \mathbb{F}_{q^{2 \ell}}\right\},
\end{aligned}
$$

Choose $t_{i}$ such that for $M_{1}=\max \left\{t_{1}, q^{\ell}-1\right\}, M_{2}=$ $\max \left\{t_{2}, q-2\right\}$ we have $S=M_{1} q+M_{2}\left(q^{\ell}-1\right) \leq q^{2 \ell+1}-q$. Then $V_{i} \subset \mathcal{L}\left(S P_{\infty}\right)$, for $i=1,2$, and for $m_{1}=\min \left\{t_{1}, q^{\ell}-\right.$ $1\}, m_{2}=\min \left\{t_{2}, q-2\right\}$ we obtain

$$
V=V_{1} \cap V_{2}=\left\{\sum_{\ell=0}^{m_{1}}\left(\sum_{j=0}^{m_{2}} a_{\ell, j} x^{j}\right) y^{\ell}: a_{\ell, j} \in \mathbb{F}_{q^{2 \ell}}\right\}
$$

and $\operatorname{dim}_{\mathbb{F}_{q^{2}}} V=\left(m_{1}+1\right)\left(m_{2}+1\right)$. The minimum distance of the code satisfies $d \geq q^{2 \ell+1}-q-S$. We have obtained the following proposition.

Proposition IV.8. Let $q$ be odd. Consider $0 \leq t_{i}, i=1,2$ satisfying that

$$
S=M_{1} q+M_{2}\left(q^{\ell}-1\right) \leq q^{2 \ell+1}-q
$$

for $M_{1}=\max \left\{t_{1}, q^{\ell}-1\right\}$ and $M_{2}=\max \left\{t_{2}, q-2\right\}$. Fixing $m_{1}=\min \left\{t_{1}, q^{\ell}-1\right\}$ and $m_{2}=\min \left\{t_{2}, q-2\right\}$ there exists $a$

$$
\left[q^{2 \ell+1}-q,\left(m_{1}+1\right)\left(m_{2}+1\right), \geq q^{2 \ell+1}-q-S ; q-1, q^{\ell}\right]
$$

recoverable code $\mathcal{C}$ over $\mathbb{F}_{q^{2 \ell}}$.

The codes arising from Proposition IV.8 have relative designed distance

$\Delta(\mathcal{C}) \leq \frac{S-\left(m_{1}+1\right)\left(m_{2}+1\right)+2-\left\lceil\frac{2\left(m_{1} m_{2}+m_{1}+m_{2}\right)+1}{q^{\ell}+q}\right\rceil}{q^{2 \ell+1}-q}$. 


\section{E. Codes from the Norm-Trace curve}

The so called Norm-Trace curve is inspired on the surjective $\mathbb{F}_{q}$-linear maps Trace and Norm from $\mathbb{F}_{q^{\ell}}$ to $\mathbb{F}_{q}$, and generalizes the Hermitian curve obtained for $\ell=2$. This curve has been object of study in [7] and [24] for construction of codes. The Norm-Trace curve is defined over $\mathbb{F}_{q^{\ell}}$ by the affine equation:

$$
y^{\frac{q^{\ell}-1}{q-1}}=x^{q^{\ell-1}}+x^{q^{\ell-2}}+\cdots+x
$$

It has genus $g=\frac{1}{2}\left(q^{\ell-1}-1\right)\left(\frac{q^{\ell}-1}{q-1}-1\right)$, only one point at infinity $P_{\infty}$ plus $q^{2 \ell-1}$ affine rational points. The functions $x$ and $y$ have pole divisor $(x)_{\infty}=\left(\frac{q^{\ell}-1}{q-1}\right) P_{\infty}$ and $(y)_{\infty}=\left(q^{\ell}-1\right) P_{\infty}$. Now consider two subgroups of the automorphism group of the function field $\mathcal{N}$ of the curve (9), given by

$$
\mathcal{H}_{1}:=\left\{(x, y) \mapsto(x+a, y) \mid \sum_{i=0}^{\ell-1} a^{q^{i}}=0, a \in \mathbb{F}_{q^{\ell}}\right\},
$$

and

$$
\mathcal{H}_{2}:=\left\{(x, y) \mapsto(x, \lambda y) \mid \lambda \in \mathbb{F}_{q^{\ell}} \text { and } \lambda^{\frac{q^{\ell}-1}{q-1}}=1\right\},
$$

so that $\# \mathcal{H}_{1}=q^{\ell-1}, \# \mathcal{H}_{2}=\frac{q^{\ell}-1}{q-1}$ and the fixed fields are $\mathcal{N}^{\mathcal{H}_{1}}=\mathbb{F}_{q^{\ell}}(y), \mathcal{N}^{\mathcal{H}_{2}}=\mathbb{F}_{q^{\ell}}(x)$ and $\mathcal{N}^{\mathcal{G}}=\mathbb{F}_{q^{\ell}}\left(x^{q^{\ell}-1}+\right.$ $\left.x^{q^{\ell}-2}+\cdots+x\right)=\mathbb{F}_{q^{\ell}}\left(y^{\frac{q^{\ell}-1}{q-1}}\right)$ by the direct product $\mathcal{G} \simeq$ $\mathcal{H}_{1} \times \mathcal{H}_{2}$. The number of rational places in $\mathcal{N}^{\mathcal{G}}$ that completely splits in the field extension $\mathcal{N} \mid \mathcal{N}^{\mathcal{G}}$ is $q-1$, then we have $n=\# \mathcal{P}=q^{\ell-1}\left(q^{\ell}-1\right)$. Following a similar construction as in section IV-D we obtain the following.

Proposition IV.9. Let $0 \leq t_{i}, i=1,2$ satisfying that

$$
S=M_{1} q+M_{2}\left(q^{\ell}-1\right) \leq q^{\ell-1}\left(q^{\ell}-1\right)
$$

for $M_{1}=\max \left\{t_{1}, \frac{q^{\ell}-1}{q-1}-2\right\}$ and $M_{2}=\max \left\{t_{2}, q^{\ell-1}-2\right\}$. Choosing $m_{1}=\min \left\{t_{1}, \frac{q^{\ell}-1}{q-1}-2\right\}$ and $m_{2}=\min \left\{t_{2}, q^{\ell-1}-\right.$ 2\} there exists a

$$
\left[n_{\ell},\left(m_{1}+1\right)\left(m_{2}+1\right), \geq n_{\ell}-S ; \frac{q^{\ell}-q}{q-1}, q^{\ell-1}-1\right]
$$

recoverable code $\mathcal{C}$ over $\mathbb{F}_{q^{\ell}}$, where $n_{\ell}=q^{\ell-1}\left(q^{\ell}-1\right)$.

This codes have relative designed distance

$$
\Delta(\mathcal{C}) \leq \frac{S-\left(m_{1}+1\right)\left(m_{2}+1\right)+2-\left\lceil\frac{2\left(m_{1} m_{2}+m_{1}+m_{2}\right)+1}{q^{\ell}+q}\right\rceil}{q^{\ell-1}\left(q^{\ell}-1\right)} .
$$

\section{GENERAL CONSTRUCTION FROM SUBGROUPS WITH NON-TRIVIAL INTERSECTION}

Here we consider non-trivial intersecting subgroups. This allows us to construct more examples.

Theorem V.1. Let $\mathcal{F} \mid \mathbb{F}_{q}$ be a function field of genus $g$. Consider $s$ non trivial subgroups $\mathcal{H}_{i}$ of the automorphism group of $\mathcal{F} \mid \mathbb{F}_{q}$ satisfying

(i) $\mathcal{G} \simeq \prod_{i=1}^{s} \mathcal{H}_{i}$ is a group; and let

(ii) \# $\left(\mathcal{H}_{i} \backslash\left(\bigcup_{j<i} \mathcal{H}_{j}\right)\right)=r_{i}$.

Let $\mathcal{P}$ be a set of $n$ places in $\mathcal{F}$ lying over rational places in the fixed field $\mathcal{F}^{\mathcal{G}}$ that are completely split in the extension $\mathcal{F} \mid \mathcal{F}^{\mathcal{G}}$. Suppose that there exists a place $P_{\infty}$ of $\mathcal{F}$ which is totally ramified in the extension $\mathcal{F} \mid \mathcal{F}^{\mathcal{G}}$ and let $Q_{\infty}^{(i)}$ be the unique place in $\mathcal{F H}^{\mathcal{H}_{i}}$ lying under $P_{\infty}$. Suppose there exists functions $z_{i}, w_{i}, i=1, \ldots, s$, such that

(iii) $z_{i} \in \mathcal{F}^{\mathcal{H}_{i}}, \operatorname{supp}\left(\left(z_{i}\right)_{\infty}\right)=\left\{Q_{\infty}^{(i)}\right\}$;

(iv) $w_{i} \in \mathcal{F} \backslash \mathcal{F}^{\mathcal{H}_{i}}, \operatorname{supp}\left(\left(w_{i}\right)_{\infty}\right)=\left\{P_{\infty}\right\}$;

(v) $w_{i}: P^{\mathcal{H}_{i}} \rightarrow \mathbb{F}_{q}$ is injective for each $i=1, \ldots, s$.

For each $i=1, \ldots, s$ let $t_{i} \geq 1$ be such that

$$
V_{i}:=\left\{\sum_{\ell=0}^{r_{i}-1}\left(\sum_{j=0}^{t_{i}} a_{\ell j}^{(i)} z_{i}^{j}\right) w_{i}^{\ell} \in \mathcal{F} \mid a_{\ell j}^{(i)} \in \mathbb{F}_{q}\right\}
$$

is contained in $\mathcal{L}\left((n-d) P_{\infty}\right)$ for some $1 \leq d \leq n$ and let $V=\bigcap_{i=1}^{s} V_{i} \subset \mathcal{L}\left((n-d) P_{\infty}\right)$. If $\operatorname{dim}_{\mathbb{F}_{q}}(V)>0$, then there exists an

$$
\left[n, \operatorname{dim}_{\mathbb{F}_{q}}(V), \geq d ; r_{1}, r_{2}, \ldots, r_{s}\right] \text {-recoverable code. }
$$

Proof. The proof uses the same ideas as in Theorem IV.1. Let $V=\bigcap_{i=1}^{s} V_{i} \subset \mathcal{L}\left((n-d) P_{\infty}\right)$ and consider the following map

$$
\begin{aligned}
e_{\mathcal{P}}: \quad V & \rightarrow \mathbb{F}_{q}^{n} \\
f & \mapsto e_{\mathcal{P}}(f)=\left(f\left(P_{1}\right), \ldots, f\left(P_{n}\right)\right),
\end{aligned}
$$

where $\mathcal{P}=\left\{P_{1}, \ldots, P_{n}\right\}$. The linear code $e_{\mathcal{P}}(V)$ is contained in the algebraic geometry code $C_{\mathcal{L}}\left(\sum_{i}^{n} P_{i},(n-d) P_{\infty}\right)$, so it has minimum distance at least $d$. This follows from the fact that the minimum distance of $e_{\mathcal{P}}(V)$ is larger than or equal to the minimum distance of $C_{\mathcal{L}}\left(\sum_{i}^{n} P_{i},(n-d) P_{\infty}\right)$. By the bound on Equation (5), the code $C_{\mathcal{L}}\left(\sum_{i}^{n} P_{i},(n-d) P_{\infty}\right)$ has minimum distance at least $d$. By definition the code has length $n$ and $\operatorname{dimension} \operatorname{dim}_{\mathbb{F}_{q}}(V)$. Now, we deal with recoverability. Assume $P \in \mathcal{P}$ is a rational place, let $Q_{i}=P \cap \mathcal{F}^{\mathcal{H}_{i}}$ be the only place in $\mathcal{F}^{\mathcal{H}_{i}}$ lying under $P$, and $\mathcal{P}_{i} \subset \mathcal{P}$ be the set of places in $\mathcal{F}$ over $Q_{i}$ for $i=1, \ldots, s$. Then $P \in \mathcal{P}_{i}$ and $\# \mathcal{P}_{i}=\# \mathcal{H}_{i}$ for $i=1, \ldots, s$ by definition of the set $\mathcal{P}$. Consider $\mathcal{Q}_{i}=\mathcal{P}_{i} \backslash\left(\bigcup_{j<i} \mathcal{P}_{j}\right)$ for any $i=1, \ldots, s$. Now, $\# \mathcal{Q}_{i}=r_{i}$ and the sets $\mathcal{Q}_{i}$ are pairwise disjoint. Using a similar approach as in Theorem IV.1, for an $f \in V_{i}$ one can show that $f(P)$ can be repaired by $\mathcal{Q}_{i}$. 
We notice that with a slight change on hypothesis of Theorem V.1 we can construct codes with a different recoverability.

Corollary V.2. With the same notation as in Theorem V.1, changing the hypothesis (ii) by

(ii') there exists $m \geq 1$ such that

$$
1 \leq \#\left(\mathcal{H}_{i} \cap\left(\bigcup_{j \neq i} \mathcal{H}_{j}\right)\right) \leq m
$$

with $\# \mathcal{H}_{i}=r_{i}+m$;

we also have the existence of an

$$
\left[n, \operatorname{dim}_{\mathbb{F}_{q}}(V), \geq d ; r_{1}, r_{2}, \ldots, r_{s}\right] \text {-recoverable code. }
$$

Proof. The proof is similar to the one in Theorem V.1, we only need to deal with recoverability. Assume $P \in \mathcal{P}$ is a rational place, let $Q_{i}=P \cap \mathcal{F}^{\mathcal{H}_{i}}$ be the only place in $\mathcal{F}^{\mathcal{H}_{i}}$ lying under $P$, and $\mathcal{P}_{i} \subset \mathcal{P}$ be the set of places in $\mathcal{F}$ over $Q_{i}$ for $i=1, \ldots, s$. Then $P \in \mathcal{P}_{i}$ and $\# \mathcal{P}_{i}=\# \mathcal{H}_{i}$ for $i=1, \ldots, s$ by definition of the set $\mathcal{P}$. From i) we also have $\#\left(\mathcal{H}_{i} \cap\left(\bigcup_{j \neq i} \mathcal{H}_{j}\right)\right) \leq m$. For any $f \in V$, using a similar approach as in Theorem IV.1 it we are going to show that $f(P)$ can be repaired by $\mathcal{P}_{i} \backslash\{P\}$ for any $i=1, \ldots, s$. Fixing $i$ we have that $f \in V_{i}$ with $\#\left(\mathcal{P}_{i} \cap\left(\bigcup_{j \neq i} \mathcal{P}_{j}\right)<m\right.$ and $\# \mathcal{P}_{i}=m+r_{i}$, so we can choose a subset $\mathcal{Q}_{i}$ of $\mathcal{P}_{i}$ such that $\# \mathcal{Q}_{i}=\# \mathcal{P}_{i}-m=r_{i}$ and $\mathcal{Q}_{i} \cap\left(\bigcup_{j \neq i} \mathcal{P}_{j}\right)=\emptyset$. Then we can recover $f(P)$ from the set $\mathcal{Q}_{i} \subseteq \mathcal{P}_{i}$. We also have the repair sets $\mathcal{Q}_{i}$ are pairwise disjoint.

\section{A. Codes from the generalized Hermitian curve}

Following the same notation as in Subsection IV-D we work with the Generalized Hermitian curve $y^{q^{\ell}+1}=x^{q}+$ $x$ over $\mathbb{F}_{q^{2 \ell}}$ and its function field $\mathcal{S}$. In this case, let $\eta, \lambda \in \mathbb{F}_{q^{2 \ell}}$ with $\operatorname{ord}(\eta)$ and $\operatorname{ord}(\lambda)$ divisors of $q^{\ell}+1$ and $\operatorname{gcd}(\operatorname{ord}(\eta), \operatorname{ord}(\lambda))=m>1$. Consider two subgroups of the automorphism group of $\mathcal{S}$ given by

$$
\begin{gathered}
\mathcal{H}_{1}:=\left\{\sigma_{i}:(x, y) \mapsto\left(x+a, \eta^{i} y\right) \mid a^{q}+a=0\right. \\
\text { and } \left.a \in \mathbb{F}_{q^{2 \ell}}, i=0, \ldots, \operatorname{ord}(\eta)-1\right\},
\end{gathered}
$$

and

$$
\begin{aligned}
\mathcal{H}_{2}:= & \left\{\sigma_{i}:(x, y) \mapsto\left(x, \lambda^{i} y\right) \mid \lambda \in \mathbb{F}_{q^{2 \ell}},\right. \\
& i=0, \ldots, \operatorname{ord}(\lambda)-1\},
\end{aligned}
$$

so that $\# \mathcal{H}_{1}=q \cdot \operatorname{ord}(\eta), \# \mathcal{H}_{2}=\operatorname{ord}(\lambda)$, they comute and $\mathcal{G}=\mathcal{H}_{1} \mathcal{H}_{2}$ has order $\frac{q \cdot \operatorname{ord}(\eta) \operatorname{ord}(\lambda)}{m}$. The fixed fields are

$$
\mathcal{S}^{\mathcal{H}_{1}}=\mathbb{F}_{q^{2 \ell}}\left(x^{q}+x, y^{\frac{q^{\ell}+1}{\operatorname{ord}(\eta)}}\right), \quad \mathcal{S}^{\mathcal{H}_{2}}=\mathbb{F}_{q^{2 \ell}}\left(x, y^{\frac{q^{\ell}+1}{\operatorname{ord}(\lambda)}}\right),
$$

and

$$
\mathcal{S}^{\mathcal{G}}=\mathbb{F}_{q^{2 \ell}}\left(x^{q}+x, y^{\frac{q^{\ell}+1}{m}}\right)
$$

where $\mathcal{G} \simeq \mathcal{H}_{1} \times \mathcal{H}_{2}$ is the direct product. The number of rational places in $\mathcal{S}^{\mathcal{G}}$ that completely splits in the field extension $\mathcal{S} \mid \mathcal{S}^{\mathcal{G}}$ is $m\left(q^{2 \ell}-1\right) /(\operatorname{ord}(\eta) \operatorname{ord}(\lambda))$, then we have $n=\# \mathcal{P}=q^{2 \ell+1}-q$. Let $0 \leq t_{i}, i=1,2$ and consider the vector spaces

$$
\begin{aligned}
& V_{1}:=\left\{\sum_{\ell=0}^{\operatorname{ord}(\eta)-2}\left(\sum_{j=0}^{t_{1}} a_{\ell, j} y^{j \frac{a^{\ell}+1}{\operatorname{ord}(\eta)}}\right) x^{\ell}: a_{\ell, j} \in \mathbb{F}_{q^{2 \ell}}\right\}, \\
& V_{2}:=\left\{\sum_{\ell=0}^{\operatorname{ord}(\lambda)-2}\left(\sum_{j=0}^{t_{2}} a_{\ell, j} x^{j}\right) y^{\ell}: a_{\ell, j} \in \mathbb{F}_{q^{2 \ell}}\right\},
\end{aligned}
$$

Choose $t_{i}$ such that for $M_{1}=\max \left\{t_{1}, \operatorname{ord}(\lambda)-2\right\}, M_{2}=$ $\max \left\{t_{2}, \operatorname{ord}(\eta)-2\right\}$ we have

$$
\begin{aligned}
S=\max & \left\{M_{1} q \frac{q^{\ell}+1}{\operatorname{ord}(\eta)}+\left(q^{\ell}+1\right)(\operatorname{ord}(\eta)-2),\right. \\
& \left.M_{2}\left(q^{\ell}+1\right)+q(\operatorname{ord}(\lambda)-2)\right\} \leq n .
\end{aligned}
$$

Then $V_{i} \subset \mathcal{L}\left(S P_{\infty}\right)$, for $i=1,2$, and for $m_{1}=$ $\min \left\{t_{1}, \operatorname{ord}(\lambda)-2\right\}, m_{2}=\min \left\{t_{2}, \operatorname{ord}(\eta)-2\right\}$ we obtain

$$
V=V_{1} \cap V_{2}=\left\{\sum_{\ell=0}^{m_{1}}\left(\sum_{j=0}^{m_{2}} a_{\ell, j} x^{j}\right) y^{\ell}: a_{\ell, j} \in \mathbb{F}_{q^{2 \ell}}\right\}
$$

and $\operatorname{dim}_{\mathbb{F}_{q^{2}}} V=\left(m_{1}+1\right)\left(m_{2}+1\right)$. The minimum distance of the code satisfies $d \geq q^{2 \ell+1}-q-S$. We have obtained the following proposition.

Proposition V.3. Let $q$ be odd, $\eta, \lambda \in \mathbb{F}_{q^{2 \ell}}^{*}$ with $\operatorname{ord}(\eta)$ and $\operatorname{ord}(\lambda)$ divisors of $q^{\ell}+1, \operatorname{gcd}(\operatorname{ord}(\eta), \operatorname{ord}(\lambda))=m>1$ and $n=q^{2 \ell+1}-q$. Choose $t_{i}$ such that for $M_{1}=$ $\max \left\{t_{1}, \operatorname{ord}(\lambda)-2\right\}, M_{2}=\max \left\{t_{2}, \operatorname{ord}(\eta)-2\right\}$ we have

$$
\begin{aligned}
S=\max \{ & M_{1} q \frac{q^{\ell}+1}{\operatorname{ord}(\eta)}+\left(q^{\ell}+1\right)(\operatorname{ord}(\eta)-2), \\
& \left.M_{2}\left(q^{\ell}+1\right)+q(\operatorname{ord}(\lambda)-2)\right\} \leq n .
\end{aligned}
$$

For $m_{1}=\min \left\{t_{1}, \operatorname{ord}(\lambda)-2\right\}, m_{2}=\min \left\{t_{2}, \operatorname{ord}(\eta)-2\right\}$ there exists a

$$
\left[n,\left(m_{1}+1\right)\left(m_{2}+1\right), \geq n-S ; q \cdot \operatorname{ord}(\eta), \operatorname{ord}(\lambda)-m\right]
$$

recoverable code $\mathcal{C}$ over $\mathbb{F}_{q^{2 \ell}}$.

The codes arising from Proposition V.3 have relative designed distance $\Delta(\mathcal{C})$ is at most

$$
\frac{S-\left(m_{1}+1\right)\left(m_{2}+1\right)+2-\left\lceil\frac{2\left(m_{1} m_{2}+m_{1}+m_{2}\right)+1}{q \cdot \operatorname{ord}(\eta)+\operatorname{ord}(\lambda)-m+1}\right\rceil}{q^{2 \ell}-q} .
$$

Remark V.4. Every example proposed in the previous section can be used to apply the construction introduced in this section as well obtaining different parameters. Indeed it is 
sufficient to enlarge a little bit one of the two groups including elements from the second one. This is the case, for instance, when comparing examples arising from Propositions IV.8 and V.3. To this end, consider $\eta, \lambda \in \mathbb{F}_{q^{2 \ell}}^{*}$, where ord $(\eta)$ divides $q^{\ell}+1, \operatorname{ord}(\lambda)=q^{\ell}+1$ and the subgroups

$$
\begin{aligned}
\mathcal{H}_{1} & =\left\{\sigma_{i}:(x, y) \mapsto(x+a, y): a^{q}+a=0, a \in \mathbb{F}_{q^{2 \ell}}\right\} \\
\mathcal{H}_{2} & =\left\{\sigma_{i}:(x, y) \mapsto\left(x, \lambda^{i} y\right): i=0, \ldots, q^{\ell}\right\}, \\
\mathcal{H}_{3} & =\left\{\sigma_{i}:(x, y) \mapsto(x, \eta y): i=0, \ldots, \operatorname{ord}(\eta)-1\right\}, \\
\widetilde{\mathcal{H}}_{1} & =\mathcal{H}_{1} \times \mathcal{H}_{3}, \\
\widetilde{\mathcal{H}}_{2} & =\mathcal{H}_{2} .
\end{aligned}
$$

Note that $\mathcal{G}=\mathcal{H}_{1} \times \mathcal{H}_{2}=\widetilde{\mathcal{H}}_{1} \widetilde{\mathcal{H}}_{2}$ and $n=q^{2 \ell+1}-q$. Let $\left(t_{1}, t_{2}\right)=\left(q^{\ell}-1, q-2\right)$ and let $\eta \in \mathbb{F}_{q^{2 \ell}}$ be such that $\operatorname{ord}(\eta) \geq q-2$. In this case, since $\operatorname{ord}(\lambda)=q^{\ell}+1$ the $\operatorname{gcd}(\operatorname{ord}(\lambda), \operatorname{ord}(\eta))=\operatorname{ord}(\eta)$, Proposition V.3 provides an

$$
\left[n, q^{\ell}(q-1), \geq n-S^{(1)} ; q \cdot \operatorname{ord}(\eta), q^{\ell}+1-\operatorname{ord}(\eta)\right]
$$

recoverable code $\mathcal{C}_{1}$ over $\mathbb{F}_{q^{2 \ell}}$, where

$$
S^{(1)}=\left(q^{2 \ell+1}-q\right) / \operatorname{ord}(\eta)+\left(q^{\ell}+1\right)(\operatorname{ord}(\eta)-2) .
$$

By Proposition IV.8 there exists an

$$
\left[n, q^{\ell}(q-1), \geq n-S^{(2)} ; q-1, q^{\ell}\right]
$$

recoverable code $\mathcal{C}$ over $\mathbb{F}_{q^{2 \ell}}$, where $S^{(2)}=2 q^{\ell+1}-2 q^{\ell}-$ $2 q+2$. This shows that recoverable codes with same length, dimension, but different minimum distance and recoverability can be obtained from similar settings.

\begin{tabular}{|c|c|c|c|c|c|}
\hline & $n$ & $k$ & $d$ & Rec. & Prop. \\
\hline $\mathbb{F}_{q^{2}}$ & $q\left(q^{2}-1\right)$ & $q$ & $\begin{array}{c}\geq q\left(q^{2}-1\right)- \\
q\left(u t_{2}+u-t_{2}-2\right)\end{array}$ & $\begin{array}{l}q-1 \\
u-1\end{array}$ & IV.2 \\
\hline $\mathbb{F}_{q^{2}}$ & $q\left(q^{2}-1\right)$ & $\begin{array}{l}\left(t_{1}+1\right) \cdot \\
\cdot\left(t_{2}+1\right)\end{array}$ & $\geq q\left(q^{2}-1\right)-M$ & $\begin{array}{l}q-1 \\
u-1\end{array}$ & IV.4 \\
\hline $\mathbb{F}_{q^{6}}$ & $\begin{array}{c}q^{8}-q^{6} \\
+q^{5}-q^{3}\end{array}$ & $\begin{array}{l}\left(M_{1}+1\right) . \\
\cdot\left(N_{1}+1\right)\end{array}$ & $\begin{array}{c}\geq q^{8}-q^{6} \\
+q^{5}-q^{3}-S\end{array}$ & $\begin{array}{c}a-1, \\
\operatorname{ord}(\eta)-1, \\
\operatorname{ord}(\omega)-1\end{array}$ & IV.6 \\
\hline $\mathbb{F}_{q^{2 \ell}}$ & $q^{2 \ell+1}-q$ & $\begin{array}{l}\left(m_{1}+1\right) \cdot \\
\cdot\left(m_{2}+1\right)\end{array}$ & $\geq q^{2 \ell+1}-q-S$ & $\begin{array}{c}q-1 \\
q^{\ell}\end{array}$ & IV.8 \\
\hline $\mathbb{F}_{q} \ell$ & $q^{\ell-1}\left(q^{\ell}-1\right)$ & $\begin{array}{l}\left(m_{1}+1\right) . \\
\cdot\left(m_{2}+1\right)\end{array}$ & $\geq q^{\ell-1}\left(q^{\ell}-1\right)-S$ & $\begin{array}{c}\frac{q^{\ell}-q}{q-1}, \\
q^{\ell-1}-1\end{array}$ & IV.9 \\
\hline $\mathbb{F}_{q^{2 \ell}}$ & $q^{2 \ell+1}-q$ & $\begin{array}{l}\left(m_{1}+1\right) \cdot \\
\cdot\left(m_{2}+1\right)\end{array}$ & $\geq q^{2 \ell+1}-q-S$ & $\begin{array}{c}q \cdot \operatorname{ord}(\eta), \\
\operatorname{ord}(\lambda)-m\end{array}$ & V.3 \\
\hline
\end{tabular}

Table I collects the parameters of the codes constructed in the previous sections. For the meaning of the symbols we refer to the corresponding proposition. Finally, in Table II we provide parameters of codes over $\mathbb{F}_{2^{12}}$ obtained for specific choices of subgroups in Propositions IV.2, IV.4, IV.6, IV.9.

Table I

\begin{tabular}{|c|c|c|c|}
\hline Code $\mathcal{C}$ & Parameters & $\Delta(\mathcal{C})$ & Prop. \\
\hline$[19656,27, \geq 18225 ; 26,27]$ & $\begin{array}{l}u=28 \\
t_{2}=1\end{array}$ & 0.0714794 & IV.2 \\
\hline$[19656,812, \geq 18171 ; 26,27]$ & $\begin{array}{c}u=28 \\
t_{1}=28 \\
t_{2}=27\end{array}$ & 0.0327635 & IV.4 \\
\hline$[6048,6, \geq 6005 ; 2,3,6]$ & $\begin{array}{c}a=3 \\
t_{1}=2, \\
t_{2}=t_{3}=1 \\
\operatorname{ord}(\eta)=4, \\
\operatorname{ord}(\omega)=7\end{array}$ & 0.0325601 & IV.6 \\
\hline$[2184,54, \geq 2080 ; 2,27]$ & $\begin{array}{c}t_{1}=26 \\
t_{2}=1 \\
q=3 \\
\ell=3\end{array}$ & 0.0219780 & IV.8 \\
\hline$[58968,7200, \geq 655 ; 90,80]$ & $\begin{aligned} t_{1} & =89 \\
t_{2} & =79 \\
q & =9 \\
\ell & =3\end{aligned}$ & 0.865385 & IV.9 \\
\hline$[2184,81, \geq 1582 ; 12,24]$ & $\begin{array}{c}t_{1}=26 \\
t_{2}=2, \\
q=3 \\
\ell=3 \\
\operatorname{ord}(\eta)=4 \\
\operatorname{ord}(\lambda)=28\end{array}$ & 0.237180 & V.3 \\
\hline
\end{tabular}

PARAMETERS OF THE OBTAINED LOCALLY RECOVERABLE CODES.
Table II

EXAMPLES OF THE LOCALLY RECOVERABLE CODES OVER $\mathbb{F}_{2^{12}}$.

\begin{tabular}{|c|c|c|c|}
\hline Code $\mathcal{C}$ & Parameters & $\Delta(\mathcal{C})$ & Prop. \\
\hline \hline$[262080,64, \geq 253952 ; 63,4]$ & $u=5$, & \multirow{2}{*}{0.03076} & IV.2 \\
& $t_{2}=31$ & & \\
\hline & $u=13$, & & \\
& $t_{1}=13$, & 0.01524 & IV.4 \\
& $t_{2}=64$ & & \\
\hline & $a=4$ & & \\
& $t_{1}=3$, & & \\
{$[6262080,910, \geq 257152 ; 63,12]$} & $t_{2}=2$, & 0.00259 & IV.6 \\
& $t_{3}=2$ & & \\
& ord $(\eta)=5$, & & \\
& ord $(\omega)=13$ & & \\
& $t_{1}=1363$, & & \\
& $t_{2}=1022$, & 0.66630 & IV.9 \\
& $q=4$, & & \\
\hline
\end{tabular}

Table III

EXAMPLES OF THE LOCALLY RECOVERABLE CODES OVER $\mathbb{F}_{36}$.

\section{ACKNOWLEDGEMENT}

This work was partially done during the visit of the third author to the University of Perugia as Visiting Researcher (project name: "Algebraic Curves and Applications", 23/01/2019-23/02/2019) and the visit of the first author to the Federal University of Rio de Janeiro (Capes - Proex). The research of D. Bartoli was partially supported by the Italian National Group for Algebraic and Geometric Structures and their Applications (GNSAGA - INdAM). 


\section{REFERENCES}

[1] A. Barg, I. Tamo, and S. Vlădut, "Locally recoverable codes on algebraic curves," IEEE Trans. Inform. Theory, vol. 63, no. 8, pp. 49284939, 2017.

[2] D. Bartoli, M. Montanucci, and G. Zini, "Multi point AG codes on the GK maximal curve," Des. Codes Cryptogr., vol. 86, no. 1, pp. 161-177, 2018.

[3] D. Bartoli, L. Quoos, and G. Zini, "Algebraic geometric codes on many points from Kummer extensions," Finite Fields Appl., vol. 52, pp. 319335, 2018.

[4] A. S. Castellanos, and G. C. Tizziotti, "Two-point AG codes on the GK maximal curves," IEEE Trans. Inform. Theory, vol. 62, no. 2, pp. 681-686, 2016.

[5] C. Galindo, H. Fernando and C. Munuera, "Locally recoverable J-affine variety codes," Finite Fields and Their Applications, vol. 64, 101661, 2020.

[6] A. Garcia, H. Stichtenoth, and C. Xing, "On subfields of the Hermitian function field," Compos. Math., vol. 120, no. 2, pp. 137-170, 2000.

[7] O. Geil, "On codes from norm-trace curves," Finite Fields Appl., vol. 9, pp. 351-371, 2003.

[8] M. Giulietti, and G. Korchmáros, "A new family of maximal curves over a finite field," Math. Ann., vol. 343, no. 1, 229, 2009.

[9] P. Gopalan, C. Huang, H. Simitci, and S. Yekhanin, "On the locality of codeword symbols," IEEE Trans. Inform. Theory, vol. 58, no. 11, pp. 6925-6934, 2012.

[10] T. Hasegawa, S. Kondo and H. Kurusu, "A sequence of one-point codes from a tower of function fields," Des. Codes Crypt., vol. 41, pp. 251267, 2006.

[11] K. Haymaker, B. Malmskog, and G.L. Matthews, "Locally recoverable codes with availability $t \geq 2$ from fiber products of curves," Adv. Math. Commun., vol. 12, no. 2, pp. 317-336, 2018.

[12] J.W.P. Hirschfeld, G. Korchmáros, and F. Torres, Algebraic Curves over a Finite Field (Princeton Series in Applied Mathematics). Princeton, NJ, USA: Princeton Univ. Press, 2008.

[13] C. Hu, and C. Zhao, "Multi-point codes from generalized Hermitian curves," IEEE Trans. Inform. Theory, vol. 62, no. 5, pp. 2726-2736, 2016.

[14] L. Jin, H. Kan, and Y. Zhang, "Constructions of locally repairable codes with multiple recovering sets via rational function fields," IEEE Trans. Inform. Theory, vol. 66, no. 1, pp. 202-209, 2020.

[15] L. Jin, L. Ma and C. Xing, "Construction of optimal locally repairable codes via automorphism groups of rational function fields," IEEE Trans. Inform. Theory, vol. 66, no 1, pp. 210-221, 2020.

[16] L. Jian, S. Mesnager, and D. Tang, "Constructions of optimal locally recoverable codes via Dickson polynomials," Designs, Codes and Cryptography, pp. 1-22, 2020. https://doi.org/10.1007/s10623-020-00731-0.

[17] S. Kondo, T. Katagiri, and T. Ogihara, "Automorphism groups of onepoint codes from the curves $y^{q}+y=x^{q^{r}+1}$, IEEE Trans. Inf. Theory, vol. 47, no. 6, pp. 2573-2579, 2001.

[18] S. Kruglik, K. Nazirkhanova, and A. Frolov, "New bounds and generalizations of locally recoverable codes with availability," IEEE Trans. Inf. Theory, vol. 65, no. 7, pp. 4156-4166, 2019.

[19] X. Li, L. Ma, and C. Xing, "Optimal locally repairable codes via elliptic curves," IEEE Trans. Inform. Theory, vol. 65, no. 1, pp. 108-117, 2019.

[20] Y. Luo, C. Xing, and C. Yuan, "Optimal locally repairable codes of distance 3 and 4 via cyclic codes," IEEE Trans. Inform. Theory, vol. 65, no. 2, pp. 1048-1053, 2019.

[21] F. J. MacWilliams, and N. J. A. Sloane, The Theory of Error-Correcting Codes. Amsterdam. The Netherlands: North-Holland, 1977.

[22] C. Munuera, and W. Tenório, "Locally recoverable codes from rational maps," Finite Fields Appl., vol. 54, pp. 80-100, 2018.

[23] C. Munuera, W. Tenório, and F. Torres, "Locally Recoverable codes from algebraic curves with separated variables," Adv. Math. Commun. vol. 14, no. 2, pp. 265-278, 2020.

[24] C. Munuera, G. C. Tizziotti, and F. Torres, "Two-point codes on normtrace curves," in Coding Theory and Applications. Lecture Notes in Computer Science, 2008, vol. 5228, pp. 128-136, Springer, Berlin, Heidelberg.
[25] H. Stichtenoth, Algebraic function fields and codes. (Graduate Texts in Mathematics), vol. 254. Berlin, Germany: Springer, 2009.

[26] I. Tamo, and A. Barg, "A family of optimal locally recoverable codes," IEEE Trans. Inf. Theory, vol. 60, no. 8, pp. 4661-4676, 2014.

[27] I. Tamo, and A. Barg, "Bounds on locally recoverable codes with multiple recovering sets," in IEEE International Symposium on Information Theory, pp. 691-695, 2014.

[28] A. Wang, and Z. Zhang, "Repair Locality With Multiple Erasure Tolerance," IEEE Trans. Inform. Theory, vol. 60, no. 11, pp. 69796987, 2014.

Daniele Bartoli was born in Molfetta, Italy, on September 22, 1985. He received the degree in Mathematics from the University of Perugia, Italy, in September 2008 and the Ph.D. degree in Mathematics and Computer Science from the same University in 2012, with a dissertation from coding theory. Currently, he holds a tenure-track position with the Department of Mathematics and Computer Science, University of Perugia, Perugia, Italy. His research interests are in coding theory, including quantum coding, and Galois geometries.

Maria Montanucci was born in Umbertide, Italy, on September 9, 1991. She received the Master degree in Mathematics from the University of Perugia, Italy, in September 2015 and the Ph.D. degree in Mathematics and Computer Science from the University of Basilicata in 2019, with a dissertation on algebraic curves over finite fields. Currently, she is Assistant Professor in Mathematics at the Technical University of Denmark, Department of Applied Mathematics and Computer Science. Her research interests are in coding theory, including quantum coding, Galois geometries, algebraic curves over finite fields and function fields.

Luciane Quoos was born in Uruguaiana, state of Rio Grande do Sul, Brazil, in 1971. She received the B.S. degree in mathematics from UFRGS, Porto Alegre/RS, in 1993, and M.S. and Ph.D. degrees in mathematics from IMPA, Rio de Janeiro/RJ, in 1995 and 2000 respectively. In 2002, she was a post-doc researcher at the Norwegian University of Science and Technology. And, in 2016 she took a one-year sabbatical at the Università degli Studi di Perugia.

In 1998 she joined the Departamento de Matemática at UFRJ, Rio de Janeiro/RJ, where she is a currently Associate Professor. Her research interests include maximal and with many rational points curves over finite fields, Weierstrass semigroups, algebraic geometry codes and permutation polynomials. 